\title{
Bifurcation Analysis of DC Electric Power Systems for Deep Space Exploration Spacecraft
}

\author{
Marc Carbone \\ Power Management and Distribution Branch \\ NASA Glenn Research Center \\ Cleveland, Ohio 44135 \\ Email: marc.a.carbone@nasa.gov
}

\author{
Amirhossein Sajadi, Kenneth Loparo \\ Electrical Engineering and Computer Science Department \\ Case Western Reserve University \\ Cleveland, Ohio 44106 \\ Email: axs1026@case.edu, kenneth.loparo@case.edu
}

\begin{abstract}
Electric power system reliability is needed to guarantee the success of space exploration missions for manned and unmanned spacecraft. Understanding the behavior of these electric systems is essential to determine the safe operating conditions, and subsequently, prevent undesired conditions that may cause system-wide blackouts, leaving the spacecraft in a vulnerable position. This study uses bifurcation analysis to determine the behavior of DC spacecraft electric power systems to identify major causes of voltage instability.
\end{abstract}

\section{INTRODUCTION}

NASA's current focus centers on developing high power vehicles to travel into deep space. The performance and stability of the onboard electric power systems, which can be considered islanded microgrids, will play a critical role in the success of these missions. Interactions between load and source subsystems will impact power quality and potentially cause momentary blackouts, malfunctions, and premature failures [1]. Therefore maintaining stability of such systems is of great importance.

Unlike terrestrial electric power systems with bulk power generation, spacecraft and aircraft have source and load power levels that are closely matched, making them very susceptible to instability, and the dynamic behavior of such systems needs to be studied to understand the operating conditions that achieve safe and reliable operation.

The designs for spacecraft electric power systems are based on photovoltaic (PV) power generation, distributed energy storage devices, distribution lines, and various resistive, constant power and AC loads. Several types of electric power system design and control approaches have been investigated for spacecraft power systems [2], [3]. Differences in these electric power systems typically involve their schemes for voltage and power regulation. For example, batteries can be connected directly to the distribution system, or regulated through DC/DC converters. Similarly, PV systems can use sequential shunting techniques, or use more sophisticated regulation including maximum power point tracking (MPPT). Depending on the requirements of the mission, various types of loads may need to be powered.

For the application of space electric power systems, there are a few notable factors that make maintaining stability challenging. First, as vehicles travel into deep space the irradi-

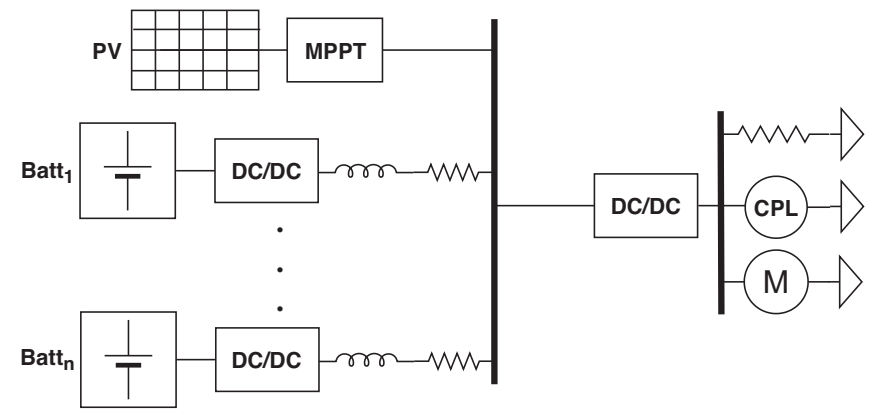

Fig. 1. Conceptual schematic diagram of DC microgrid onboard a spacecraft

ance available to PVs decreases, limiting the total generating capacity of the system. Autonomous systems currently lack the ability to reliably track PV capacity in real-time due to unexpected damage. In addition, distributed energy storage batteries must be carefully controlled to regulate bus voltage for the loads. Adjustments in load impedance, generation capacity, and degradation cause frequent changes in system stability that are difficult to monitor. Lastly, interactions between source and load subsystems may cause unexpected conditions for instability depending on the design parameters of a particular system.

This paper focuses on applications that use regulated PV and battery sources to provide power to the loads. The regulated sources will allow for improved controllability and efficiency of the power flow. A wide range of voltage regulation techniques have been used on spacecraft electric power systems. Each control implementation strongly affects the stability characteristics of the electric power system during operation. In order to understand this behavior an approximate model of the electrical distribution network is developed and analyzed to generate the nonlinear dynamics of the system. To construct this analytical model for the distribution electrical power network, individual subsystem models [1], [4], [5] were used. A conceptual model of such a DC power system with source and load subsystems is shown in Fig. 1. Based on this model, bifurcation analysis is used to calculate the operating conditions that will satisfy the stability requirements [6]-[8].

Bifurcation analysis studies how variations in system pa- 
rameters change equilibria and the qualitative behavior of the dynamical system [9]. The bifurcation point refers to an operating condition where the stability of the system changes as one or more parameters vary [10]. This study uses bifurcation analysis to establish necessary conditions for stability of a DC space electric power system. In particular, the effects of variations in power generated by solar panels, power consumed by the loads, and battery energy storage on the equilibria and voltage stability of the system are examined.

Presently, supervision of spacecraft electric power systems are achieved from ground stations on earth by human operators. Communication delays for missions beyond low earth orbit (LEO) are estimated to be up to 44 minutes round trip [11]. Due to the potential dangers of these communication latencies, many of the monitoring functions currently conducted on the ground must be implemented in autonomous software onboard the spacecraft that is capable of intelligent decisionmaking. The findings of this paper can be used to develop an approach for real-time stability monitoring of DC electric power systems for autonomous spacecraft operations. In future work, this analysis will be verified using a real hardware testbed.

\section{DC Electric Power System Model}

To create a general model of a DC microgrid for spacecraft electric power system, it is necessary to understand each component's individual dynamic behavior. The components considered here include PV sources under MPPT, parallel batteries under droop control, distribution lines, bus capacitance, loads (active, resistive, and inductive) and DC/DC isolating converters. Modeling the behavior of the power source and load subsystems is most crucial because the interactions between them defines the system's stability properties.

\section{A. PVs and Maximum Power Point Tracking}

PV sources are often connected to the electrical distribution network through a regulating device that defines the dynamic behavior of the source [12]. PVs are often controlled via MPPT through a DC/DC converter, where the objective is to maximize the power output. The PV cell can be modeled as a current source (photo-current, $I_{p h}$ ) that is dependent on the incident solar radiation (insolation) [13]. The entire cell can be modeled as a P-N junction, and therefore behaves as a diode. Practically, there is some resistance $R_{s}$ in the path of the junction. Also some of the electrons and holes recombine before reaching the electrodes at the main bus. This has the effect of shunting a part of the output current and can be modeled using a parallel resistance $R_{s h}$. Thus, a PV cell can be modeled by the equivalent circuit represented in Fig. 2. A group of connected cells are used to create the entire PV array and can be approximated by modifying the single cell model.

The diode current can be calculated as [13]:

$$
i_{D}=I_{0}\left(e^{A v_{d}}-1\right)
$$

where $I_{0}$ is the saturation current of the diode, $A=\frac{q}{\gamma k T_{e}}$, $q$ is the charge of the electron $\left(=1.6 \times 10^{-19}\right.$ coulombs $)$,

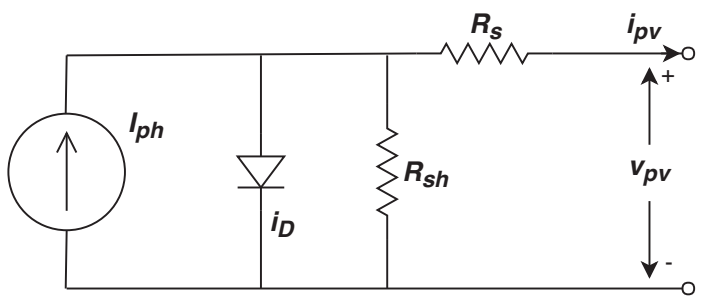

Fig. 2. Equivalent 1-diode model of a PV cell

$\gamma$ is the diode ideality factor, $k$ is Boltzmann's constant (= $1.38 \times 10^{-23} \mathrm{~J} / \mathrm{K}$ ), and $T_{e}$ is the absolute temperature. Then, the current flow through the cell is characterized by:

$$
\begin{gathered}
i_{p v}=I_{p h}-i_{D}-i_{s h} \\
i_{p v}=I_{p h}-I_{0}\left(e^{A v_{d}}-1\right)-\frac{v_{D}}{R_{s h}} .
\end{gathered}
$$

The voltage over the diode can be defined by:

$$
v_{D}=v_{p v}+i_{p v} R_{s},
$$

By substituting equation (4) into (3), the cell current can be expressed by:

$$
i_{p v}=I_{p h}-I_{0}\left(e^{A\left(v_{p v}+i_{p v} R_{s}\right)}-1\right)-\frac{v_{p v}+i_{p v} R_{s}}{R_{s h}} .
$$

Then, the single cell model can be extended to approximate the full array [14]:

$$
i_{p v}=N_{p} I_{p h}-N_{p} I_{0}\left(e^{A\left(\frac{v_{p v}}{N_{s}}+\frac{i_{p v} R_{s}}{N_{p}}\right)}-1\right)-\frac{N_{p}}{R_{s h}}\left(\frac{v_{p v}}{N_{s}}+\frac{i_{p v} R_{s}}{N_{p}}\right)
$$

where $N_{p}$ and $N_{s}$ are the number of parallel and series cells respectively.

The full PV array model of equation (6) must be solved numerically using a Newton-Raphson algorithm. Solving this equation for different photo-currents (i.e. changing solar conditions) yields the $I-V$ curves and, subsequently, $P$ - $V$ curves. The $I-V$ and $P-V$ curves are shown in Figs. 3, and 4.

Many algorithms have been implemented for the MPPT, including perturb and observe ( $\mathrm{P} \& \mathrm{O})$, hill-climb, and incremental conductance (IncCond) [15] to name a few. In general, these algorithms successfully condition the PV to operate near the maximum power point, unaffected by the behavior of the other subsystems. Therefore, the maximum power point (peak of the P-V curves) for each level of solar irradiation are used to model the PV array as a constant power source.

\section{B. Batteries and Droop Control}

The distributed batteries are responsible for maintaining bus voltage within acceptable limits. The effects of other subsystems, such as the PV array and loads, creates challenges for the batteries to maintain the proper bus voltage. In this study, droop control, a common method in microgrid applications, is used to regulate the bus voltage. It should be noted that other types of control schemes can also be used, and these may 


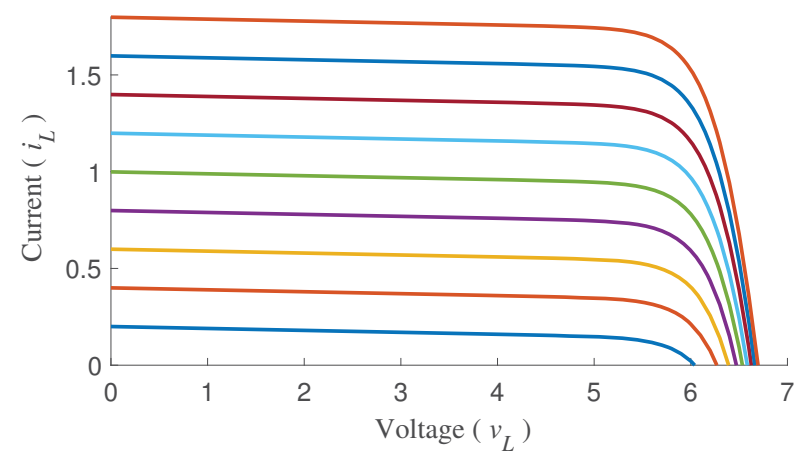

Fig. 3. The $I-V$ characteristics of a solar array

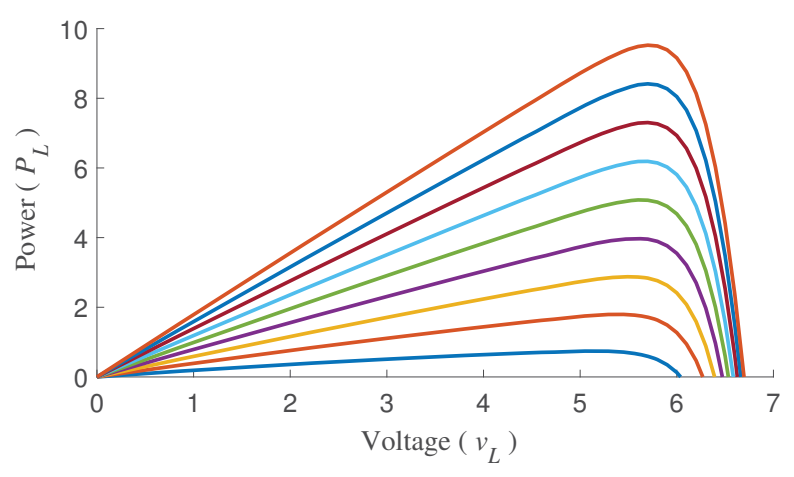

Fig. 4. The $P-V$ characteristics of a solar array

have a significant impact on the system's dynamic behavior and therefore, its stability.

The main concept of droop control is to perform load sharing while accounting for the generating capacity of each load so that no source becomes overloaded. This paper uses the model for distributed batteries operating under droop control from [1]. In this model, a battery connected to the bus through a power converter interface can be viewed as an ideal voltage source with a controlled (virtual) droop resistance, $V_{i}$ and $R_{v i}$ respectively. The source connects to the system over a distribution line, that can be modeled using a resistance, $R_{l i}$, and inductance $L_{l i}$. In general, $R_{v i} \gg R_{l i}$, and, thus, it may be assumed $R_{v i} \approx R_{v i}+R_{l i}$.

For $n$ batteries in parallel, the reference voltage $V_{\text {ref }}$ is set for all sources such that

$$
V_{r e f} \approx V_{1} \approx V_{2} \approx \cdots \approx V_{n}
$$

Then, it can be assumed that under normal droop conditions

$$
\frac{R_{v_{1}}}{L_{l_{1}}} \approx \frac{R_{v_{2}}}{L_{l_{2}}} \approx \ldots \approx \frac{R_{v_{n}}}{L_{l_{n}}}
$$

so that a single term can be used to represent the approximated equivalent resistance and inductance by taking the average of each of the source parameters

$$
\frac{R_{d}}{L_{d}}=\frac{1}{n} \sum_{j=1}^{n} \frac{R_{v_{j}}}{L_{l_{j}}}
$$

This approximation helps reduce the $n$ differential equations to a single differential equation. The equivalent (approximate) model simplifies the stability analysis with only minor effects on accuracy.

For a single droop controlled source $k$, the dynamics can be modeled using the differential equation [1]

$$
\frac{d i_{k}}{d t}=\frac{1}{L_{l_{k}}}\left(V_{\text {ref }}-v_{\text {bus }}\right)-\frac{R_{d_{k}}}{L_{t_{k}}} .
$$

To reduce the behavior of the entire microgrid to a single differential equation, the total current provided by all of the droop controlled sources can be defined as

$$
i_{s} \approx i_{1}+i_{2}+\cdots+i_{n}
$$

Then the differential equation for the sum of sources becomes

$$
\frac{d i_{s}}{d t}=\left(\sum_{j=1}^{n} \frac{1}{L_{l_{j}}}\right)\left(V_{\text {ref }}-v_{\text {bus }}\right)-\sum_{j=1}^{n} \frac{R_{d_{j}}}{L_{l_{j}}} i_{j} .
$$

Next, using the approximation from equation (8) we can assume

$$
\frac{d i_{s}}{d t} \approx\left(\sum_{j=1}^{n} \frac{1}{L_{l_{j}}}\right)\left(V_{r e f}-v_{b u s}\right)-\frac{R_{d}}{L_{d}} i_{s} .
$$

Equation (13) can be further simplified by multiplying both sides of the equation by

$$
L_{e q}=\frac{1}{\sum_{j=1}^{n} \frac{1}{L_{l_{j}}}}
$$

that produces

$$
\frac{d i_{s}}{d t}=\frac{1}{L_{e q}}\left[\left(V_{r e f}-v_{b u s}\right)-R_{e q} i_{s}\right]
$$

where

$$
R_{e q}=R_{d} \frac{L_{e q}}{L_{d}}
$$

The droop controlled batteries can be modeled using equation (15), which can be viewed as a voltage source in series with an inductor and resistor.

\section{Constant Power Loads}

The constant power loads (CPL) are modeled as current sinks, where the constant current consumed is equal to the CPL power divided by the CPL voltage. An isolating DC/DC converter connects the loads to the main bus to regulate the source subsystem voltage to the CPL voltage. It is assumed that the power input into the converter approximately equals the power output. Therefore the DC/DC converter can be modeled as a CPL as long as the input voltage is greater than or equal to the required voltage of the loads $V_{0}$. If the input voltage falls below $V_{0}$, the converter acts as a passive load. Fig. 5 shows the behavior of the DC/DC converter. In addition, in [16], [17] active and passive damping is shown to be useful 


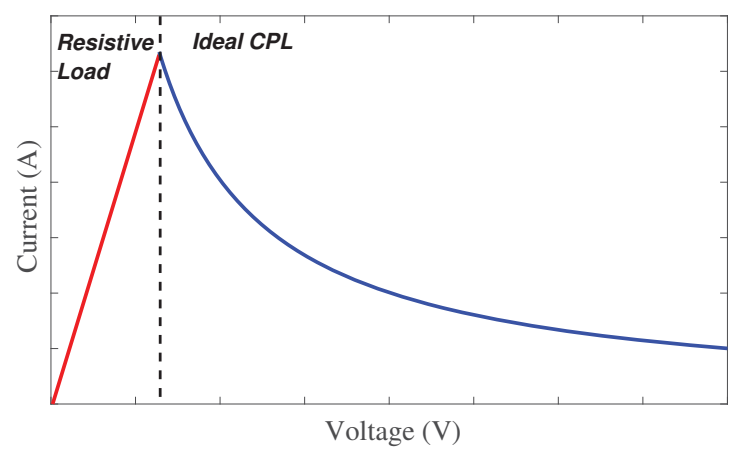

Fig. 5. Dynamic behavior of the DC/DC converter

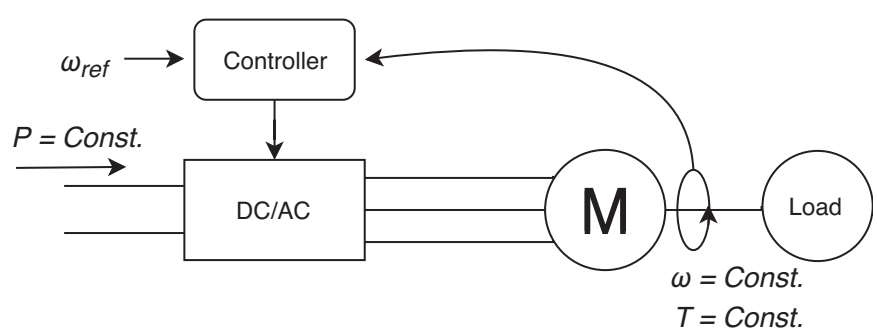

Fig. 6. DC/AC inverter generating constant power load characteristics

in controlling the converter output for poorly behaved loads. In order to maintain the simplicity of the analysis, only the CPL behavior of the converters is considered and the behavior of the DC/DC converters can be approximated as

$$
i(v)=\frac{P}{v} .
$$

Other types of spacecraft loads require AC power such as motors and pumps. Under tightly regulated control, these loads also behave as CPLs [16]. In the case of an electric motor, the angular velocity is accurately controlled by the DC/AC inverter (as shown in Fig. 6). Due to the linear relationship between speed and torque, a given operating speed corresponds to a single value of torque. Power is equal to the product of the speed and torque, and electric motors can be considered as constant power loads [18].

\section{StABility FrameWORK}

Now that each subsystem model is developed, an approximate model can be written for the entire spacecraft microgrid. Integrating the components of the system generates the circuit shown in Fig. 7. The dynamic equations for the full system are

$$
\begin{gathered}
\frac{d i_{s}}{d t}=\frac{1}{L_{e q}}\left(V_{\text {ref }}-v_{\text {bus }}-R_{e q} i_{s}\right) \\
\frac{d v_{\text {bus }}}{d t}=\frac{1}{C}\left(i_{s}-\frac{v_{\text {bus }}}{R}-\frac{P}{v_{\text {bus }}}\right)
\end{gathered}
$$

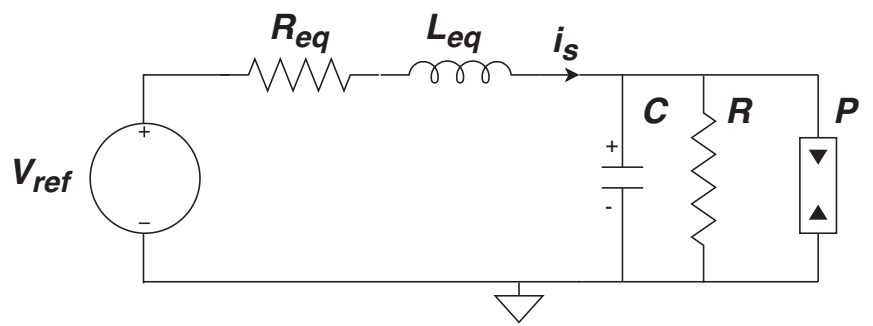

Fig. 7. Equivalent model for droop controlled DC microgrid

where the net power $P$, is defined as the difference between the power consumed and the power generated by the PV arrays:

$$
P=P_{L}-P_{P V}
$$

For the sake of clarity, $P$ represents the amount of power that exceeds the maximum generation capabilities of the PV arrays, and this power must be supplied from the remaining battery power. Next, $C$ represents the lumped sum of the input capacitances from the isolating DC/DC converters as well as the sum of the capacitor banks attached to each bus.

To obtain the equilibrium points of this system, the right hand side (RHS) of the differential equations should be set equal to zero. Solving for the two fixed points yields

$$
\begin{aligned}
& {\left[I_{1}^{*}, v_{\text {bus }_{1}}^{*}\right]=\left(\frac{V_{\text {ref }}-v_{\text {bus }_{1}}^{*}}{R_{d}}, \frac{R V_{\text {ref }}-a}{2\left(R_{d}+R\right)}\right)} \\
& {\left[I_{2}^{*}, v_{\text {bus }_{2}}^{*}\right]=\left(\frac{V_{\text {ref }}-v_{\text {bus }_{2}}^{*}}{R_{d}}, \frac{R V_{\text {ref }}+a}{2\left(R_{d}+R\right)}\right)}
\end{aligned}
$$

where $a=\sqrt{R^{2} V_{r e f}^{2}-4 P R R_{d}\left(R_{d}+R\right)}$.

The stability of each fixed point can be determined by evaluating the eigenvalues of the of the Jacobian matrix $(J)$ of the system.

$$
J=\left[\begin{array}{cc}
-\frac{R_{e q}}{L_{e q}} & -\frac{1}{L_{e q}} \\
\frac{1}{C} & \frac{1}{C}\left(\frac{P}{v_{\text {bus }_{i}}^{* 2}}-\frac{1}{R}\right)
\end{array}\right]
$$

The first fixed point, $\left[I_{1}^{*}, v_{b u s_{1}}^{*}\right]$ produces eigenvalues of opposite signs resulting in an unstable saddle-node equilibrium. The second fixed point, $\left[I_{2}^{*}, v_{b u s_{2}}^{*}\right]$ produces a stable fixed point when the determinant of $J$ is positive $(\Delta>0)$, and the trace of $J$ is negative $(\tau<0)$. Solving both inequalities results in stability when $C>\frac{L_{e q}}{R_{e q}^{2}}$. The case where $C \leq \frac{L_{e q}}{R_{e q}^{2}}$ is discussed in the next section. Under this design consideration, the maximum net power that can be supported by the system is

$$
P<\frac{R V_{r e f}^{2}}{4 R_{e q}\left(R_{e q}+R\right)}=P_{\max }
$$

where $P_{\max }$ is the maximum power that can be supported by the batteries. This is the minimum power value for which the parameter $a$ is real and a meaningful solution exists. The bifurcation diagram in Fig. 8 shows the behavior of the two fixed points as a function of net power $P$. The following electrical network parameters are used for the simulation: 


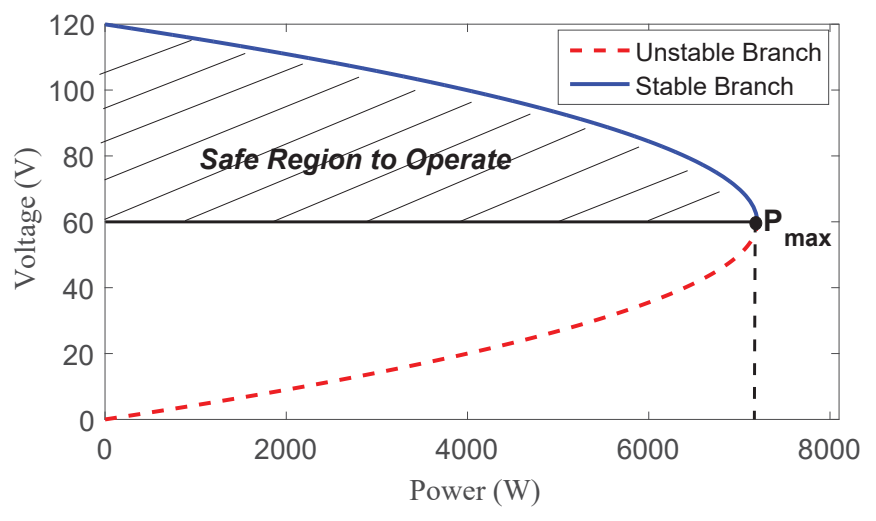

Fig. 8. Bifurcation diagram of the approximate model

$V_{\text {ref }}=120(V), R=1000(\Omega), R_{e q}=0.5(\Omega), L_{e q}=$ $500(\mu H), C=3(m F)$.

A voltage bifurcation diagram that relates voltage to active power is shown in Fig. 8, also known as a nose curve. This curve has two main branches; the top branch, shown with a solid blue line, indicates all stable solutions whereas the bottom branch, a red dashed line, indicates all unstable solutions. The point where the two branches meet is known as the bifurcation point or voltage instability point corresponding to the loading level at which the two solutions coalesce into one [19]. This curve determines the stability characteristics of the electric power system for a given set of operating conditions, even when small disturbances occur. Moreover, the bifurcation curve reveals what operational limits must be avoided to prevent instability. For this bifurcation curve, it can be seen that the electric power system considered here is unstable when $P>P_{\max }$. For example, increasing the CPL load $P_{L}$ so that $P_{L}-P_{P V}>P_{\max }$ causes the system to be unstable. Such a condition can result in large voltage oscillations or even total voltage collapse of the system.

The system operator (human or computer) can use this information as an analytical tool to ensure that the electrical power system is operating within the stable region. Methods such as parameter estimation [20] can be used with sensor measurements to accurately approximate the network variables at any point during a mission, then using the stability framework, judgments could be made as to how close the system is to a point of instability. Periodic updates of the stability status is a highly beneficial resource to the power controller in order to prevent overloading and other causes of voltage failure.

\section{Simulation RESUlts}

Next the bifurcation can be used to gain an understanding of the sensitivity to different system parameters and variables. Observing several "what-if" scenarios acts as a contingency analysis study for events that could occur in the electrical power network. In this section, four credible scenarios are addressed. The first three scenarios involve a static phenomenon known as a saddle-node bifurcation. Whereas the fourth scenario results in a dynamic phenomenon known as a Hopf bifurcation.

\section{A. Effect of PV Generation}

Assuming that there are two equally sized solar arrays on the spacecraft, the effect of a fault causing an outage of one of the arrays can be observed in Fig. 9. In the bifurcation diagram, voltage is displayed as a function of the excess power. The loss of a single array would cause the fixed point to move along the bifurcation curve (from point A) to the right for the exact amount of PV power loss due to the failure (to point B). As a result, the system experiences a voltage drop. This event causes the power that needs to be supported by the battery outputs to increase to restore the voltage. The example in Fig. 9 shows the effect of losing half of the total PV generation capability in the system. Here the power $P$ remains below $P_{\max }$ and hence, the system will remain stable. Otherwise, the system would be come unstable and appropriate corrective actions such as load shedding can be used to maintain or restore the stability.

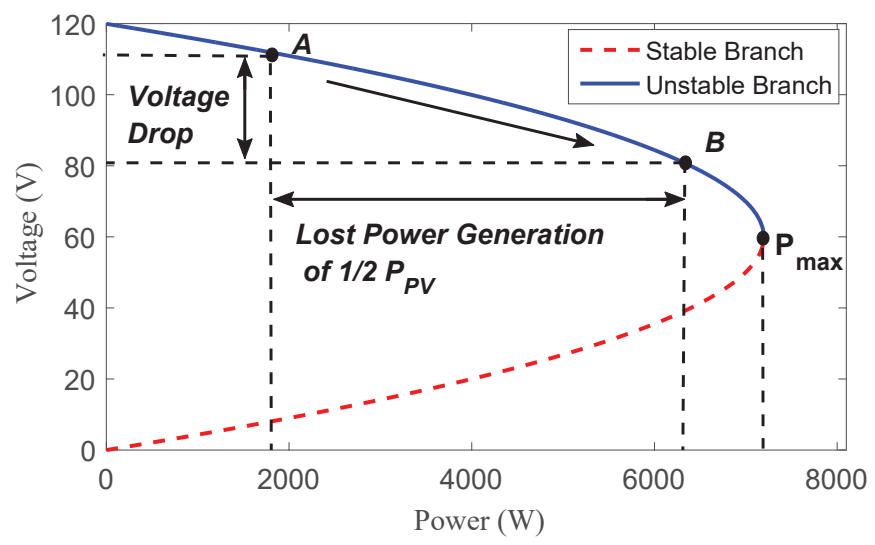

Fig. 9. Electrical power system operating point before and after PV array failure

\section{B. Effect of Load Resistance}

Another parameter that affects the stability of the electrical network is the load resistance $R$. Equation (24) shows that for $R \gg R_{e q}$ the stability limit does not change significantly for small changes in $R$. If the resistive load becomes quite small (around two orders of magnitude of $R_{e q}$ ), then the bifurcation curve changes as shown in Fig. 10. This example shows the resistance change from $R_{1}=1000(\Omega)$ to $R_{2}=2(\Omega)$. The lack of resistive load in the system with respect to the equivalent droop resistance causes the voltage to drop from point A to point B. Given that the system is still stable when operating below $P_{\max }$, it is able to restore its voltage through a corrective action such as load shedding (operating point moves from point B to point C). This introduces an important feature in that the stability of the system is not the only operating condition that needs to be considered. Each of the CPLs in the electrical network has a minimum voltage that is required for operation. Therefore $v_{\text {bus }}$ must be greater than 
the minimum allowable CPL voltage, even if the system is stable. For example, if the minimum required CPL voltage is $V_{0}=90(V)$, as shown in Fig. 10, then the operating voltage must remain above $V_{0}$ for the CPLs to function properly.

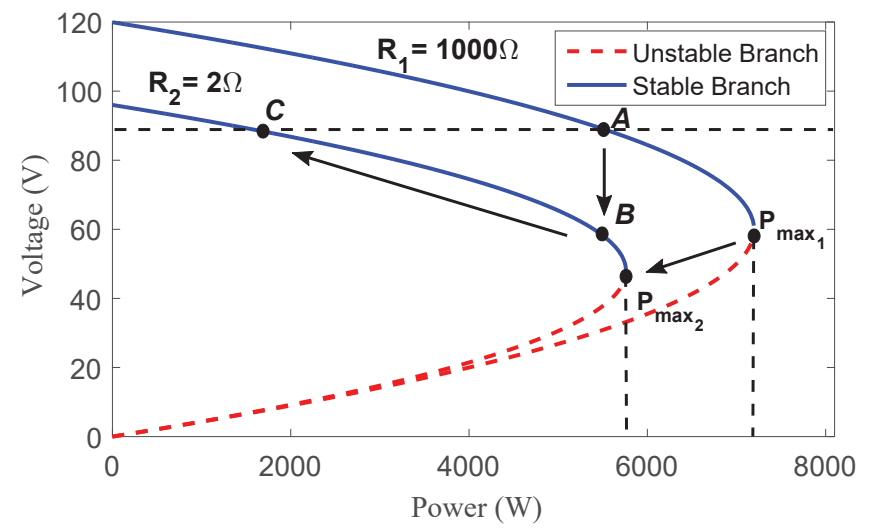

Fig. 10. Bifurcation curves with changing load resistance $R$

\section{Effect of Droop Resistance}

Next, the effect of $R_{e q}$ on the bifurcation curve is shown in Fig. 11. The first bifurcation curve shows equivalent droop resistance $R_{e q 1}=0.5$, and the second with $R_{e q 2}=0.1$. Note that as the equivalent droop resistance decreases, the maximum operating load increases drastically from $7.2 \mathrm{~kW}$ (point A) to $18 \mathrm{~kW}$ (point B). Therefore, it is beneficial for the power electrical system controller to minimize the equivalent droop resistance of the energy storage devices. This will allow the sources to support the largest load demands.

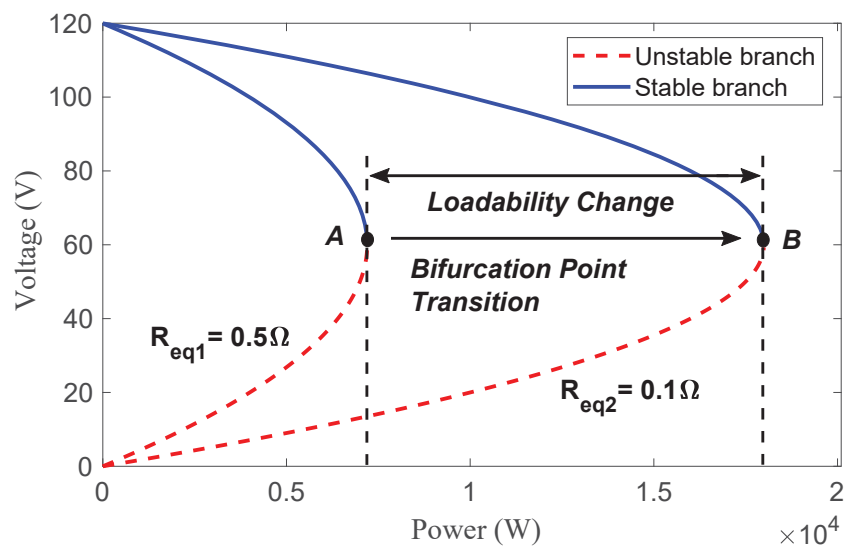

Fig. 11. Bifurcation curves with changing equivalent droop resistance $R_{e q}$

\section{Effect of Capacitance, Inductance, and Droop Resistance}

Thus far, three scenarios have been addressed where total capacitance, equivalent inductance, and droop resistance satisfy $C>\frac{L_{e q}}{R_{e q}^{2}}$. This condition guarantees the determinant of the Jacobian matrix remains positive $(\Delta>0)$ and its trace remains negative $(\tau<0)$. Such condition results in a saddlenode bifurcation, that occurs when the number of equilibrium points changes as a parameter changes [21].

An alternative condition implies the total capacitance, equivalent inductance, and droop resistance satisfy $C \leq \frac{L_{e q}}{R_{e q}^{2}}$. This condition indicates that the system no longer maintains $\Delta>0$ and $\tau<0$ for the entire range of real fixed points. Therefore, the region of stability of the electrical power system may shrink, and as a result, is stable for a narrower range of load demand. This is due to a dynamic phenomenon known as a Hopf bifurcation. The Hopf bifurcation condition occurs when the number of equilibrium points remains unchanged, but the eigenvalues of the Jacobian matrix are purely imaginary [21].

Analyzing the Jacobian matrix for the system with $C=$ $3(m F)$ shows that there are two eigenvalues in the left half plane for the entire range of $P$ examined. Therefore a saddlenode bifurcation occurs at $P \approx 7.2(k W)$. However, the eigenvalues of the system with $C=1(\mathrm{mF})$ move toward the right half plane as the value of $P$ increases and they eventually cross the imaginary axis at $P \approx 6.4(\mathrm{~kW})$ when $\tau \geq 0$, where the Hopf bifurcation occurs. The bifurcation diagram of the system with the value of total capacitance of $C=1(\mathrm{mF})$ is shown in Fig. 13. This diagram reveals how the bifurcation point moves from $P_{\max } \approx 7.2(\mathrm{~kW})$ to $P_{\max } \approx 6.4(\mathrm{~kW})$, reflecting a decrease in the ability of the system to maintain the power delivery to the system's load. Therefore it is important to design the electrical power system with sufficient bus capacitance so that $C>\frac{L_{e q}}{R_{e q}^{2}}$ across the entire operating range of the system.

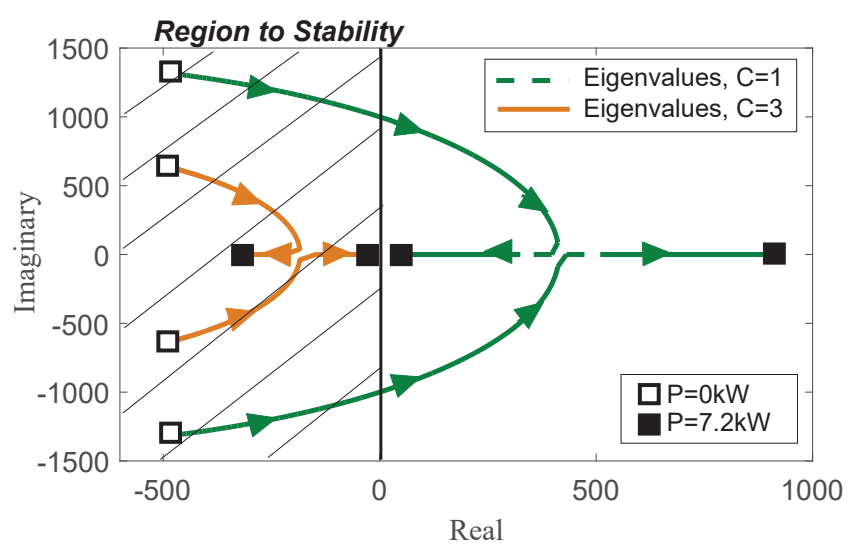

Fig. 12. Eigenvalues of the Jacobian as a function of net power $P$

To summarize, each parameter of the electrical power system plays an important role in the voltage stability characteristics of the system. Stability analysis is an important element in design as well as the online supervision of an electric power system. Taking the stability into consideration during the design process will ensure that the potential serviceable load is maximized and that contingencies such as PV array failures can be mitigated and effectively managed. 


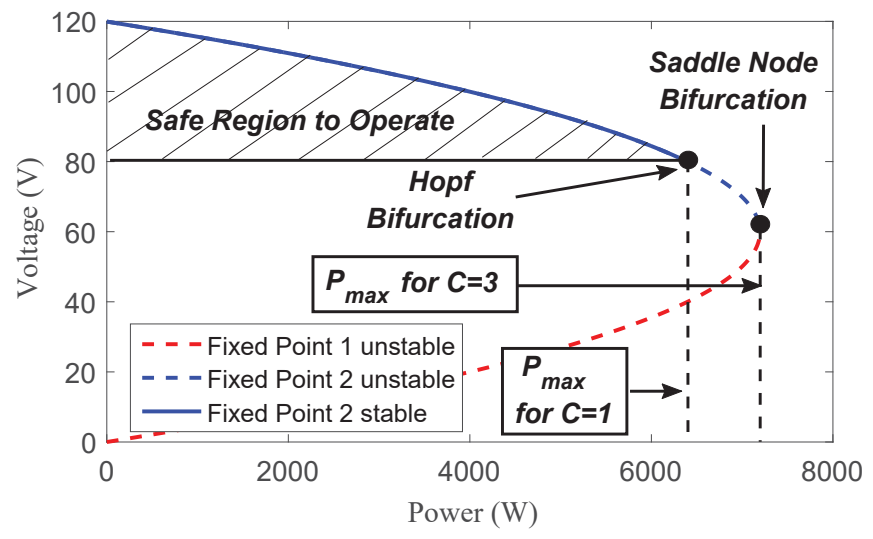

Fig. 13. Bifurcation curve with decreased capacitance $C=1(m F)$

\section{CONCLUSION}

This paper identifies the need for tracking the stability of DC spacecraft electric power systems. The subsystem dynamic behavior was used to create a mathematical model for each electrical power component. Using the subsystem models, an approximate model for the entire spacecraft electric power system was developed. From this overall model, the fixed points of the system were computed and their stability analyzed. The bifurcation study shows how stability changes with respect to the adjustment of system parameters and operating conditions. Understanding the bifurcation process allows the electric power system controller to avoid unstable points during operation. Instabilities can lead to wide-scale blackouts due to voltage oscillations and voltage collapse, which put the success of any space mission at risk. Therefore it is critical to accurately predict the bifurcations of spacecraft power systems. The next step in this effort is to test the validity of the model against a real hardware testbed. Once the model is verified to a reasonable extent, the stability framework can be developed into a software package that may provide useful information to the electric power system controller.

\section{REFERENCES}

[1] A. P. N. Tahim, D. J. Pagano, E. Lenz, and V. Stramosk, "Modeling and stability analysis of islanded dc microgrids under droop control," IEEE Transactions on Power Electronics, vol. 30, no. 8, pp. 4597-4607, 2015.

[2] D. B. McKissock, "Development and use of the space computer code for analyzing the space station electrical power system," 2018.

[3] R. May and K. A. Loparo, "The use of software agents for autonomous control of a dc space power system," in 12th International Energy Conversion Engineering Conference, 2014, p. 3860.

[4] J. Liu, W. Zhang, and G. Rizzoni, "Robust stability analysis of dc microgrids with constant power loads," IEEE Transactions on Power Systems, vol. 33, no. 1, pp. 851-860, 2018.

[5] N. H. van der Blij, L. M. Ramirez-Elizondo, M. T. Spaan, and P. Bauer, "A state-space approach to modelling dc distribution systems," IEEE Transactions on Power Systems, vol. 33, no. 1, pp. 943-950, 2018.

[6] N. H. van der Blij, L. M. Ramirez-Elizondo, P. Bauer, and M. T. Spaan, "Design guidelines for stable dc distribution systems," in DC Microgrids (ICDCM), 2017 IEEE Second International Conference on. IEEE, 2017, pp. $279-284$.

[7] J. Schiffer, R. Ortega, A. Astolfi, J. Raisch, and T. Sezi, "Conditions for stability of droop-controlled inverter-based microgrids," Automatica, vol. 50, no. 10, pp. 2457-2469, 2014.
[8] W. Yi and D. J. Hill, "Stability analysis of all inverter-interfaced generation systems," in 2018 Power Systems Computation Conference (PSCC). IEEE, 2018, pp. 1-7.

[9] C. Ç. Karaaslanl, "Bifurcation analysis and its applications," in Numerical simulation-from theory to industry. InTech, 2012.

[10] S. H. Strogatz, Nonlinear dynamics and chaos: with applications to physics, biology, chemistry, and engineering. CRC Press, 2018.

[11] J. Frank, L. Spirkovska, R. McCann, L. Wang, K. Pohlkamp, and L. Morin, "Autonomous mission operations," in 2013 IEEE Aerospace Conference. IEEE, 2013, pp. 1-20.

[12] D. Chen, L. Xu, and L. Yao, "Dc voltage variation based autonomous control of dc microgrids," IEEE transactions on power delivery, vol. 28, no. 2 , pp. 637-648, 2013.

[13] A. Abusorrah, M. M. Al-Hindawi, Y. Al-Turki, K. Mandal, D. Giaouris, S. Banerjee, S. Voutetakis, and S. Papadopoulou, "Stability of a boost converter fed from photovoltaic source," Solar energy, vol. 98, pp. 458471, 2013.

[14] F. Ding and K. A. Loparo, "Dynamic modeling and stability analysis of grid-connected and autonomous distributed generation system," in Innovative Smart Grid Technologies Conference (ISGT), 2015 IEEE Power \& Energy Society. IEEE, 2015, pp. 1-5.

[15] T. Esram and P. L. Chapman, "Comparison of photovoltaic array maximum power point tracking techniques," IEEE Transactions on energy conversion, vol. 22, no. 2, pp. 439-449, 2007.

[16] M. K. AL-Nussairi, R. Bayindir, S. Padmanaban, L. Mihet-Popa, and P. Siano, "Constant power loads (cpl) with microgrids: Problem definition, stability analysis and compensation techniques," Energies, vol. 10, no. 10, p. 1656, 2017.

[17] M. Ashourloo, A. Khorsandi, and H. Mokhtari, "Stabilization of dc microgrids with constant-power loads by an active damping method," in Power Electronics, Drive Systems and Technologies Conference (PEDSTC), 2013 4th. IEEE, 2013, pp. 471-475.

[18] A. Emadi, A. Khaligh, C. H. Rivetta, and G. A. Williamson, "Constant power loads and negative impedance instability in automotive systems: definition, modeling, stability, and control of power electronic converters and motor drives," IEEE Transactions on vehicular technology, vol. 55, no. 4, pp. 1112-1125, 2006.

[19] T. J. Overbye, I. Dobson, and C. L. DeMarco, "Qv curve interpretations of energy measures for voltage security," IEEE Transactions on Power Systems, vol. 9, no. 1, pp. 331-340, 1994.

[20] P. Young, "Parameter estimation for continuous-time modelsa survey," Automatica, vol. 17, no. 1, pp. 23-39, 1981.

[21] C.-W. Tan, M. Varghese, P. Varaiya, and F. Wu, "Bifurcation and chaos in power systems," Sadhana, vol. 18, no. 5, pp. 761-786, 1993. 